\title{
Posterior vertebral column resection in early onset spinal deformities
}

\author{
D. Jeszenszky • D. Haschtmann · F. S. Kleinstück • \\ M. Sutter · A. Eggspühler $\cdot$ M. Weiss $\cdot$ T. F. Fekete
}

Received: 21 January 2013/Revised: 9 June 2013/Accepted: 18 July 2013/Published online: 25 August 2013

(C) Springer-Verlag Berlin Heidelberg 2013

\begin{abstract}
Purpose Early onset spinal deformities (EOSD) can be life-threatening in very young children. In the growing spine, surgical intervention is often unavoidable and should be carried out as soon as possible. A deformed section of the spine not only affects the development of the remaining healthy spine, but also that of the chest wall (which influences pulmonary function), the extremities and body balance. Posterior vertebral column resection (PVCR) represents an effective surgical solution to address such problems. However, reports in the literature concerning PVCR are mostly limited to its use in adolescents or adults. The purpose of this study was to illustrate our experience with PVCR in EOSD and to describe the surgical technique with respect to the unique anatomy of young children.

Materials and methods Four children [mean age 3.7 (range 2.5-5.2) years] with severe spinal deformity underwent PVCR through a single approach. Multimodal intraoperative monitoring was used in all cases. Surgery included one stage posterior circumferential resection of one vertebral body along with the adjoining intervertebral discs and removal of all posterior elements. A transpedicular screw-rod system was used for correction and stabilisation. Fusion was strictly limited to the resection site,
\end{abstract}

D. Jeszenszky and D. Haschtmann have contributed equally.

D. Jeszenszky · D. Haschtmann $(\bowtie) \cdot$ F. S. Kleinstück ·

M. Sutter · A. Eggspühler · T. F. Fekete

Schulthess Clinic, Spine Center, Lengghalde 2, 8008 Zürich,

Switzerland

e-mail: dezsoe.jeszenszky@kws.ch

URL: www.schulthess-clinic.ch

M. Weiss

Childrens Hospital, University of Zürich, Zürich, Switzerland allowing for later conversion into a growing rod construct at the remaining spine, if necessary. Relevant data were extracted retrospectively from patient charts and long spine radiographs.

Results The mean operation time was 500 (range 463-541) min, with an estimated blood loss of 762 (range 600-1,050) $\mathrm{ml}$. Mean follow-up time was 6.3 (range 3.5-12.4) years. After PVCR, the mean Cobb angle for scoliosis was reduced from $69^{\circ}$ (range 50-99 ${ }^{\circ}$ ) to $29^{\circ}\left(5-44^{\circ}\right)$ and the sagittal curvature (kyphosis) from $126^{\circ}\left(87-151^{\circ}\right)$ to $61^{\circ}\left(47-75^{\circ}\right)$. The mean correction of scoliosis was $57 \%\left(18-92^{\circ}\right)$ and of kyphosis, $51 \%$ $\left(44-62^{\circ}\right)$. There were no spinal cord-related complications. In three patients, spinal instrumentation for growth guidance (fusion less growing rod technique) was applied. Two patients had complications: one patient had a complication of anesthesia, halo pin failure, and revision surgery with extension of the instrumentation cranially due to loss of correction; the second patient had a postoperative infection, which required plastic reconstructive measures.

Conclusion PVCR appears to be an effective technique to treat severe EOSD. There are important differences in its use in young children when compared with older patients. In patients with EOSD, additional surgical procedures are often necessary during growth, and hence non-fusion instrumentation beyond the vertebral resection site is advantageous, as it permits spinal growth and the later addition of fusion.

Keywords Early onset spinal deformity · Early onset scoliosis · Posterior vertebral column resection . Growing rod instrumentation - Surgical technique · Multimodal intraoperative monitoring · Surgical complications - Paediatric spine surgery 


$\begin{array}{ll}\text { Abbreviations } \\ \text { VCR } & \text { Vertebral column resection } \\ \text { PVCR } & \text { Posterior vertebral column resection } \\ \text { PSO } & \text { Pedicle subtraction osteotomy } \\ \text { SPO } & \text { Smith-peterson osteotomy } \\ \text { MIOM } & \text { Multimodal intraoperative monitoring } \\ \text { EOSD } & \text { Early onset spinal deformity } \\ \text { CPR } & \text { Cardiopulmonary resuscitation } \\ \text { PJK } & \text { Proximal junctional kyphosis } \\ \text { EOS } & \text { Early onset scoliosis }\end{array}$

\section{Introduction}

Progressive severe early onset spinal deformities ${ }^{1}$ (EOSD) in younger children can be life-threatening if pulmonary development is impaired. EOSD by definition encompasses kyphotic, scoliotic or combined deformities with an onset before the age of five [1]. EOSD is frequently found in complex congenital syndromes [2, 3]. Conservative treatment is often inadequate and surgical intervention becomes unavoidable. As development in early childhood is very rapid, surgical correction can become urgent [4]. If treatment is delayed, the deformity may result in neurological compromise and may affect the development of otherwise normal regions of the spine (including the chest wall), the extremities, and the balance of the body [5]. Severe chest wall deformity at a young age results in hypoplastic lungs with an irreversible impairment of pulmonary function $[6,7]$.

Vertebral column resection (VCR) has become established as a standard surgical procedure to correct severe deformities that cannot be adequately dealt with by using alternative techniques such as (extended) PSO and multiple SPOs. VCR is defined as a circumferential resection of at least one vertebra with all its anterior and posterior elements along with the adjoining intervertebral discs. It was first described by Bradford [8] as a combined approach and later established as a single posterior approach by Suk et al. [9]. However, the published reports are mostly limited to adolescent and adult patients [8-17]. There are few publications and limited data on VCR in the growing spine [18], and, to the best of our knowledge, no reports have dealt exclusively with EOSD. In the detailed report by Lenke et al. [12], describing 43 cases of posterior VCR (PVCR), the emphasis was clearly on adolescent and adult deformity.

\footnotetext{
$\overline{1}$ We prefer to use the abbreviation EOSD rather than EOS (early onset scoliosis) because scoliosis is not the only type of deformity seen in these patients (see Table 1).
}

The surgical treatment of EOSD differs from that of the deformities of skeletally more mature patients (adolescents and adults) for many reasons. Unlimited extension of the instrumented fusion above and below the site of the threecolumn osteotomy (VCR), in the interests of safety/stability, is not recommended. The subsequent arrest of growth in the operated area would lead to permanently impaired pulmonary function and an unacceptably short trunk, and would pose the risk of crankshaft phenomenon.

The purpose of this study was to illustrate our experience with PVCR, with spinal fusion limited to the resection site, in a series of four patients with severe EOSD. We describe the surgical technique, especially in relation to the unique anatomical features of young children, and present data on the extent of deformity correction and the rate and type of complications.

\section{Materials and methods}

\section{Patients}

Between the years 2000 and 2008 (minimum 4-year follow-up), four patients up to the age of 5 years underwent posterior vertebral column resection (PVCR) by one surgeon (first author). All children presented with a documented rapid progression of the spinal deformity, including trunk asymmetry and/or noticeably reduced physical activity due to respiratory insufficiency (patients BA, TJ, ML). One patient had had to be fed with a PEG tube due to malnutrition secondary to dyspnea and exhaustion (patient $\mathrm{TJ}$ ). The indication for surgery was respiratory failure and a rigid severe angular kyphoscoliotic or kyphotic deformity (Table 1). In three children (patient BA, ML, BJ), halo traction was applied preoperatively. Clinical examination was carried out with neurological assessment and documentation of spinal curvature, balance and posture (in children who were able to walk). There were no abnormal neurological findings. Preoperative surgical planning with radiologic evaluation included traction and/or bolster hyperextension films as well as long spine plain X-rays, computed tomography and magnetic resonance imaging. The appropriate segment at the apex of the curve, the number of vertebrae to be excised and the additional osteotomies required were determined.

Surgical technique

A conventional radiolucent orthopaedic surgery table was used, with the patients being placed in a prone position on specially made Maquet-like adjustable modular foam-pillows that acted as a frame. Children under halo-gravity traction were positioned with a traction weight of $1-3 \mathrm{~kg}$. 
Table 1 Radiographic measures of the coronary and sagittal curves in four patients with EOSD (Cobb angles)

\begin{tabular}{|c|c|c|c|c|c|c|}
\hline & BA & ML & FJL & TJ & Mean & SD \\
\hline \multicolumn{7}{|c|}{ Scoliosis main curve (coronal Cobb angle) } \\
\hline Preoperative curve $\left({ }^{\circ}\right)$ & 99 & 69 & 59 & 50 & 69 & 21 \\
\hline Initial postop curve $\left({ }^{\circ}\right)$ & 44 & 26 & 5 & 41 & 29 & 18 \\
\hline Correction initial postop (\%) & 56 & 62 & 92 & 18 & 57 & 30 \\
\hline Latest follow-up curve $\left({ }^{\circ}\right)$ & 33 & 9 & 5 & 8 & 14 & 13 \\
\hline Latest correction (\%) & 67 & 87 & 92 & 84 & 82 & 11 \\
\hline \multicolumn{7}{|c|}{ Scoliosis compensatory curve (coronal Cobb angle) } \\
\hline Preoperative curve $\left(^{\circ}\right)$ & 38 & 38 & 54 & 18 & 37 & 15 \\
\hline Initial postop curve $\left({ }^{\circ}\right)$ & 23 & 9 & 18 & 15 & 16 & 6 \\
\hline Correction initial postop $(\%)$ & 39 & 76 & 67 & 17 & 50 & 27 \\
\hline Latest follow-up curve $\left(^{\circ}\right)$ & 7 & 5 & 11 & 5 & 7 & 3 \\
\hline Latest correction $(\%)$ & 82 & 87 & 80 & 72 & 80 & 6 \\
\hline \multicolumn{7}{|l|}{ Kyphosis (sagittal Cobb angle) } \\
\hline Preoperative curve $\left({ }^{\circ}\right)$ & 142 & 124 & 87 & 151 & 126 & 28 \\
\hline Initial postop curve $\left({ }^{\circ}\right)$ & 54 & 69 & 47 & 75 & 61 & 13 \\
\hline Correction initial postop (\%) & 62 & 44 & 46 & 50 & 51 & 8 \\
\hline Latest follow-up curve $\left(^{\circ}\right)$ & 62 & 43 & 44 & 69 & 55 & 13 \\
\hline Latest correction (\%) & 56 & 65 & 49 & 54 & 56 & 7 \\
\hline \multicolumn{7}{|l|}{ Lordosis (sagittal Cobb angle) } \\
\hline Preoperative curve $\left({ }^{\circ}\right)$ & 84 & 79 & 44 & 61 & 67 & 18 \\
\hline Initial postop curve $\left({ }^{\circ}\right)$ & 42 & 37 & 45 & 47 & 43 & 4 \\
\hline Correction initial postop (\%) & 50 & 53 & -2 & 23 & 31 & 26 \\
\hline Latest follow-up curve $\left(^{\circ}\right)$ & 79 & 46 & 42 & 47 & 54 & 17 \\
\hline Latest correction (\%) & 6 & 42 & 5 & 23 & 19 & 17 \\
\hline Follow-up (years) & 12.4 & 5.2 & 4.2 & 3.5 & 6.3 & 4.1 \\
\hline
\end{tabular}

Mean values are indicated in bold

After skin incision, a supra-periosteal preparation was carried out followed by verification of the correct levels using fluoroscopy. Pedicle screws were then placed using a free-hand technique with entry points for the pedicle screws being determined using anatomical landmarks. Thus, 22 gauge needles were inserted into the pedicles, and followed by fluoroscopic control in anteroposterior and lateral views. The pedicles were prepared using a $1.5 \mathrm{~mm}$ drill, and pre-bent $\mathrm{K}$-wires were inserted, followed by further fluoroscopy. Based on the fluoroscopic images, fine adjustments of the proposed pedicle screw trajectory were made with a 2.0 or $2.5 \mathrm{~mm}$ drill. The size of the pedicle screws was dependent on the diameter of the pedicles; in all patients, $3.5 \mathrm{~mm}$ screws were used. Wide laminectomies and costo-transversectomies on one or both sides were performed in all cases. This provided a capacious working area with optimal visualization of the dural sac during the correction manoeuvers. It also allowed for safe circumferential preparation of the vertebral body and protection of the segmental and major vessels. The exiting nerve roots were marked with vessel loops. A single nerve root may be killed in the thoracic area between Th3 and Th9. However, a deficiency of Th4 and/or Th5 root function may lead to sensory loss in the mammary area, which may cause problems later in life (e.g. with breast-feeding). The existence of distinctive tissue layers, due to the thick periosteum, facilitated the resection of the vertebral body. First the intervertebral discs above and below the vertebral body were resected. Bony resection did not necessarily require the use of a burr which is typical of an eggshell procedure carried out in adults. In contrast, the vertebral body could often be removed in one piece with preservation of the posterior wall. Before final removal of the vertebral body, a unilateral rod was applied, to provide temporary stabilisation and prevent translational movements. Above and below the vertebral resection site, two blocks of several spinal segments were instrumented with pedicle screws. This ensured optimal control and an even distribution of forces during the correction maneuvers (in situ bending, translation, rod rotation and compression/distraction). At this stage, during which the spine was substantially destabilized, it was essential to avoid translation, subluxation and dural impingement. The correction had to be performed slowly and carefully, with continuous inspection of the dural sac/spinal cord and nerve roots. In addition, careful and repeated circumferential palpation of the dura was necessary to assess any impingement, over-distraction or excessive shortening.

The length of the spinal cord should ideally remain constant, with the theoretical pivot point of correction being at the given spinal level and within the confines of the spinal cord. This usually involved shortening of the posterior elements, and in most cases, lengthening anteriorly. Anterior column support was achieved either by simple compression (bone-on-bone contact) or by inserting a cage (patient TJ, Harms cage) or structural graft (patient $\mathrm{TL}$, from bone bank).

Bony fusion was facilitated by the use of morcellized autologous bone graft from the vertebra and ribs that were removed. Resection of the rib head along with the PVCR cannot be avoided. On the concave side, the rib may have to be shortened as it may otherwise impinge on the spinal cord during correction. However, extended rib resection on the convex side and rib-osteotomy on the concave side (thoracoplasty) should be avoided wherever possible, to prevent undesirable rib fusions or stiffening of the chest wall. Based on intraoperative findings and preoperative planning, additional osteotomies were carried out in three patients (patient BA, TJ, FJL). At times, the decision to perform resection at more than one level had to be made during surgery, depending on the correction already achieved and possible alerts from multimodal intraoperative monitoring (MIOM). As a final step, fluoroscopic images were obtained in anteroposterior and lateral projections, to ensure that adequate spinal alignment and implant positioning had been achieved. Relevant data such 
as operation time, blood loss, change of MIOM signals, intraoperative surgical complications and complications of anesthesia were recorded.

\section{Postoperative management}

In young children-just as in adults-pedicle screw instrumentation provides sufficient stability to allow patients to be mobilized without any external orthosis. The children in the present study did not need to be encouraged to get up and move, and they typically mobilized themselves as soon as the wound pain had subsided. Physiotherapy was carried out mainly for the improvement of lung function. No patient required chest tubing following surgery.

Follow-up assessments were carried out at 8 weeks, 6 months, and 1 year after surgery and included radiographic and clinical assessment as well as documentation of complications and any further surgeries. Depending on the stage of spinal growth, further follow-ups were planned at 6-month to 1-year intervals. If a significant residual deformity was present, conversion to a growing rod construct with repeated distraction was performed (patient BA, ML, TJ). Radiographs were analyzed, with curve measurements being made in the coronal and sagittal planes using the Cobb method.

\section{Results}

Patients

The demographic, clinical and surgical data for the four patients are summarized in Table 2. The mean age at the time of surgery was 3.7 (range $2.5-5.2$ ) years. The mean follow-up time was 6.3 (range 3.5-12.4) years. Two children suffered from syndromic EOSD: one with congenital deformity in Arthrogryposis multiplex congenita (AMC; patient BA) and one with Goldenhar syndrome (Pierre Robin Sequence; patient FJL). In two patients, the syndrome was undefined (patients ML and TJ) (Fig. 1). In one patient, (patient TJ) PVCR was carried out as a secondary procedure, following a posterior decompression and $360^{\circ}$ release at $\mathrm{T} 5 / 6$ and instrumentation of $\mathrm{T} 2-\mathrm{T} 9$ with postoperative loss of correction. Preoperative halo-gravity traction was applied in three patients (patients BA, ML, TJ; Table 2). Two of these learnt to walk during the course of the preoperative traction (patients BA, ML). In all patients, PVCR was carried out at one level only for correction of the deformity. The average operation time was 500 (range 463-541) min, with an estimated blood loss of 762 (range 600-1,050) ml. All patients were operated under continuous MIOM, carried out by the experienced

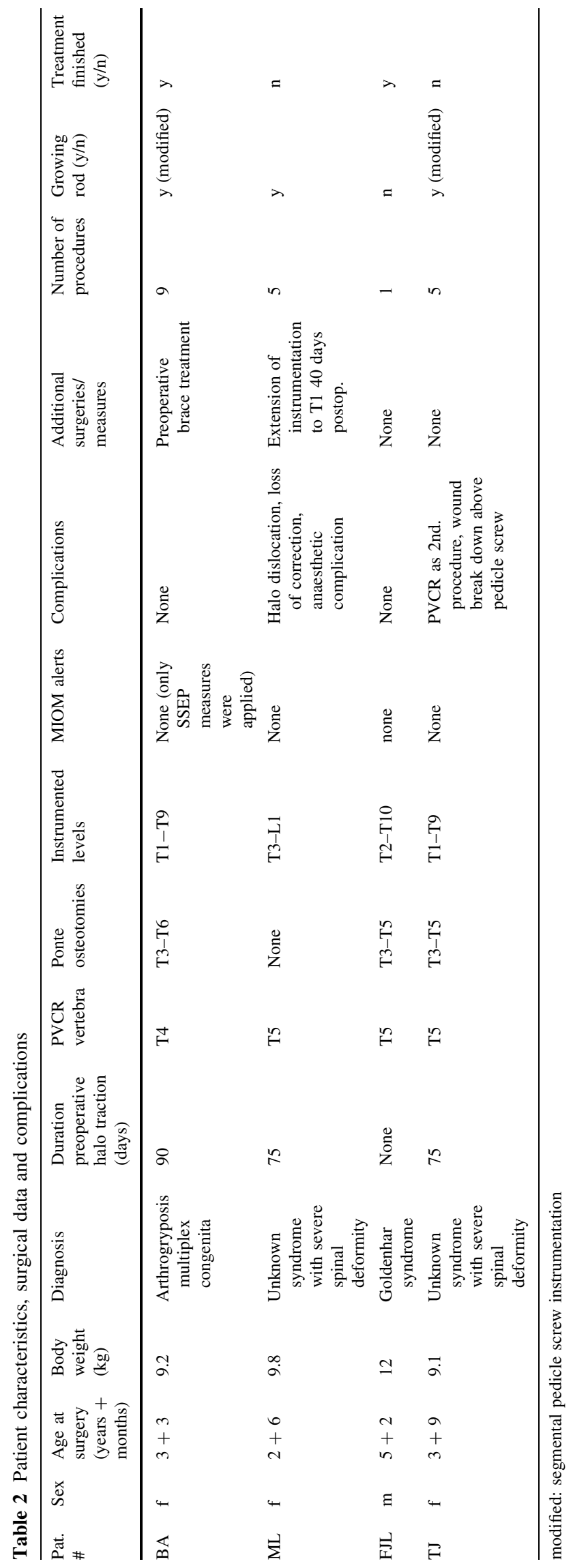




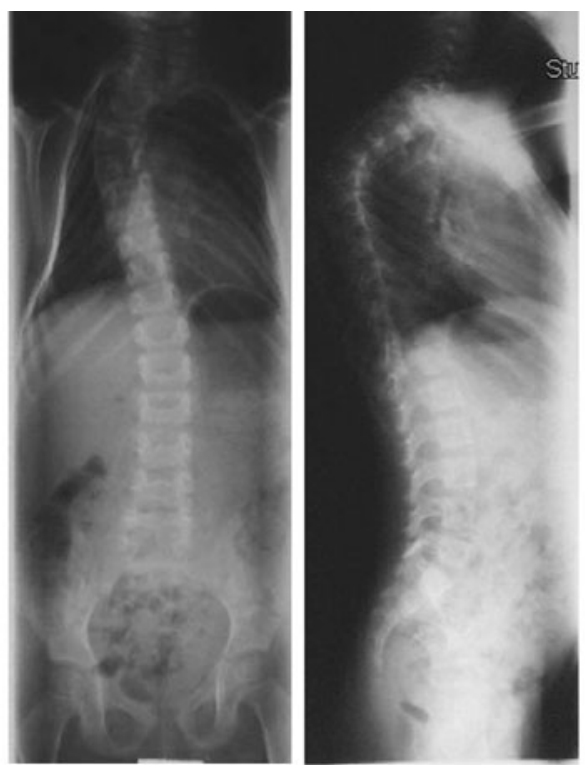

BA
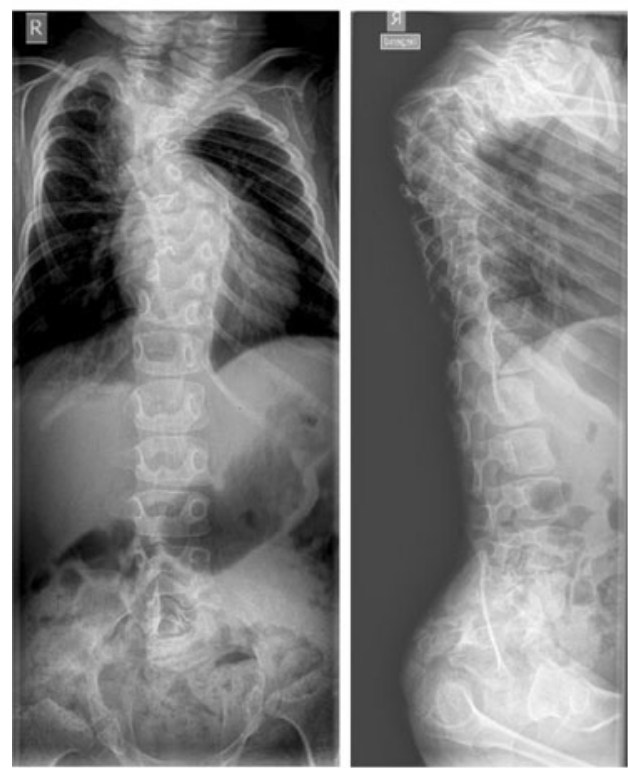

FJL

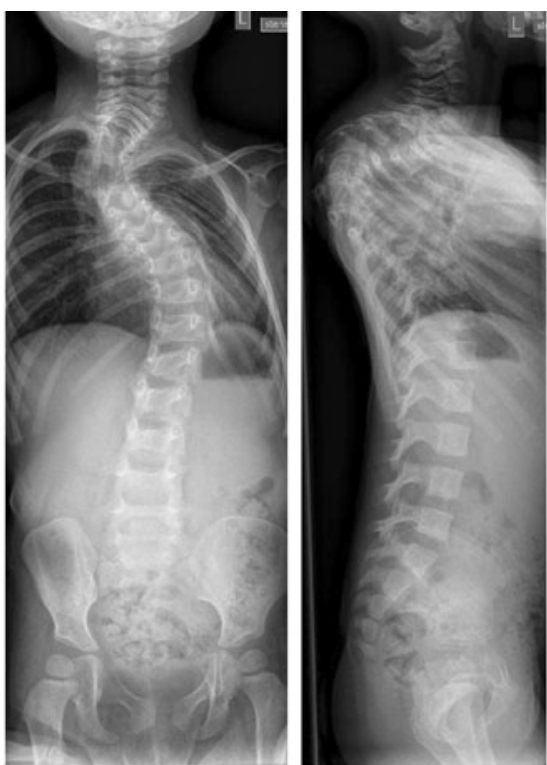

$\mathrm{ML}$
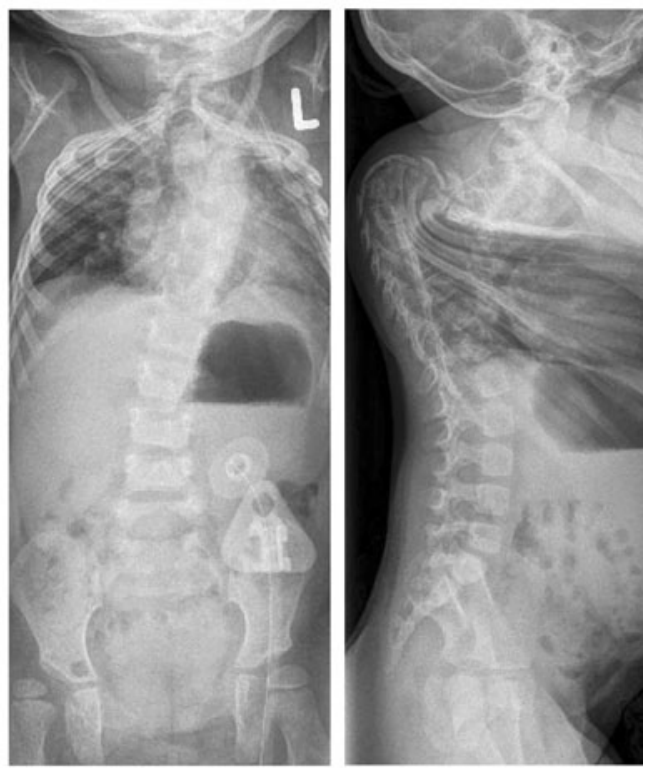

TJ

Fig. 1 Radiographs of four patients with severe EOSD [patients BJ (AMC), ML (unknown syndrome), FJL (Goldenhar syndrome), TJ (unknown syndrome)]

neurophysiologists, which included motor evoked potentials (MEP) and somatosensory evoked potentials (SSEP) [19]. There were no postoperative neurological deficits. In three patients, growth guidance techniques were applied (patients BA, ML, TJ; Table 2). In two of these (patients BA, TJ), a modified growth preservation construct was used, with segmental non-fusion pedicle screw instrumentation and repeated segmental distraction, while in one patient (patient ML), a growing rod construct was used [20]. In the latter patient, the growing rod was removed later to allow the unrestricted growth (Fig. 2d). In all patients with preoperative dyspnea at rest, a significant improvement was observed after surgery.

Radiological results

\section{Primary curves}

Table 1 shows the extent of deformity correction in the anteroposterior and sagittal planes for the individual patients. With PVCR, the mean preoperative scoliosis angle of the major curve (Cobb angle) was reduced from 
Fig. 2 Clinical and

radiographic course of a severe early onset thoracic

kyphoscoliotic deformity (a).

The little girl (2.5 years, ML) with an unknown syndrome underwent posterior vertebral column resection of $\mathrm{T} 5$ at the age of 2 years and 6 months, after 75 days of halo traction. Non-fusion posterior

instrumentation was carried out from T3 to L1. Postoperatively, proximal junctional kyphosis developed rapidly and the instrumention was extended to T1 (b). Note the marked changes in symmetry of the chest and posture (c). Segmental instrumentation was replaced by a double growing rod construct 3.6 years later (d) followed by multiple distractions. The curvature improved during continued growth, and the instrumention could be partially removed, i.e. shortened (d). The motion segments were still functioning, as no fusion was intended in this area. Clinical aspect at the latest follow-up, aged 7 years (e). Close followup is neccessary to detect any deterioration, especially during the prepubertal growth spurt

\section{a}
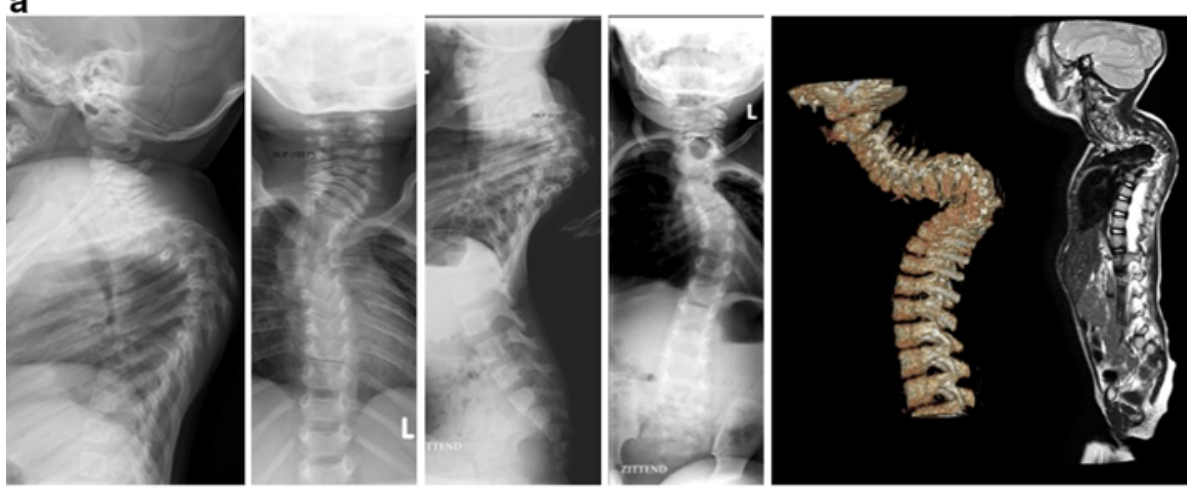

\section{b}
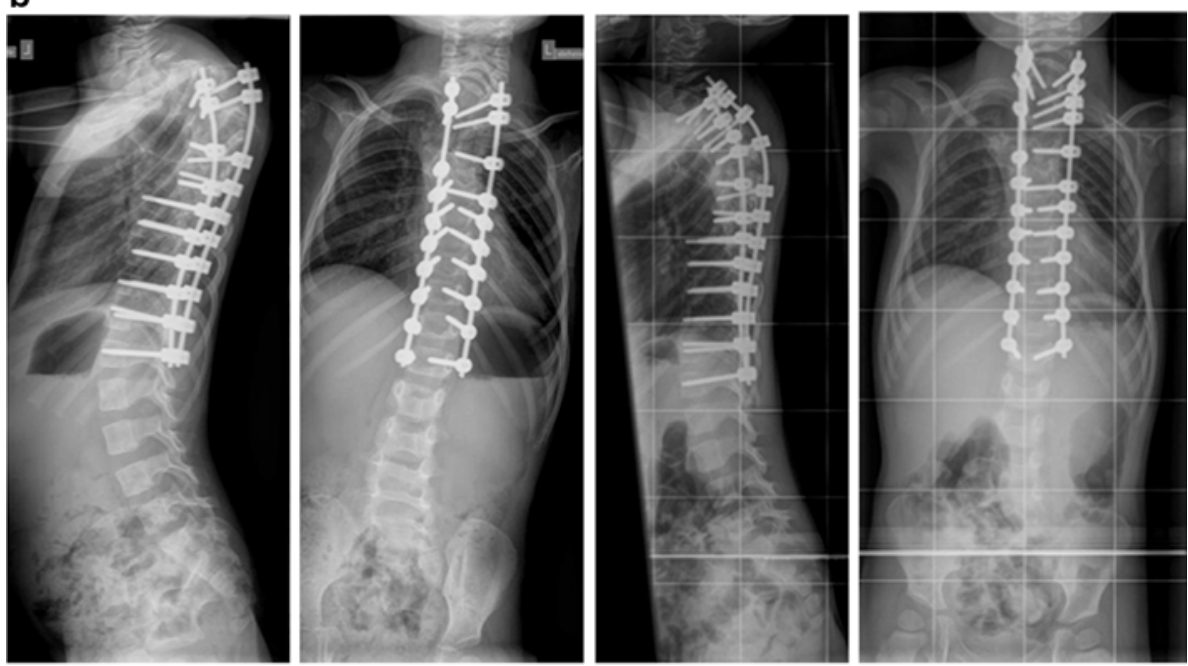

C
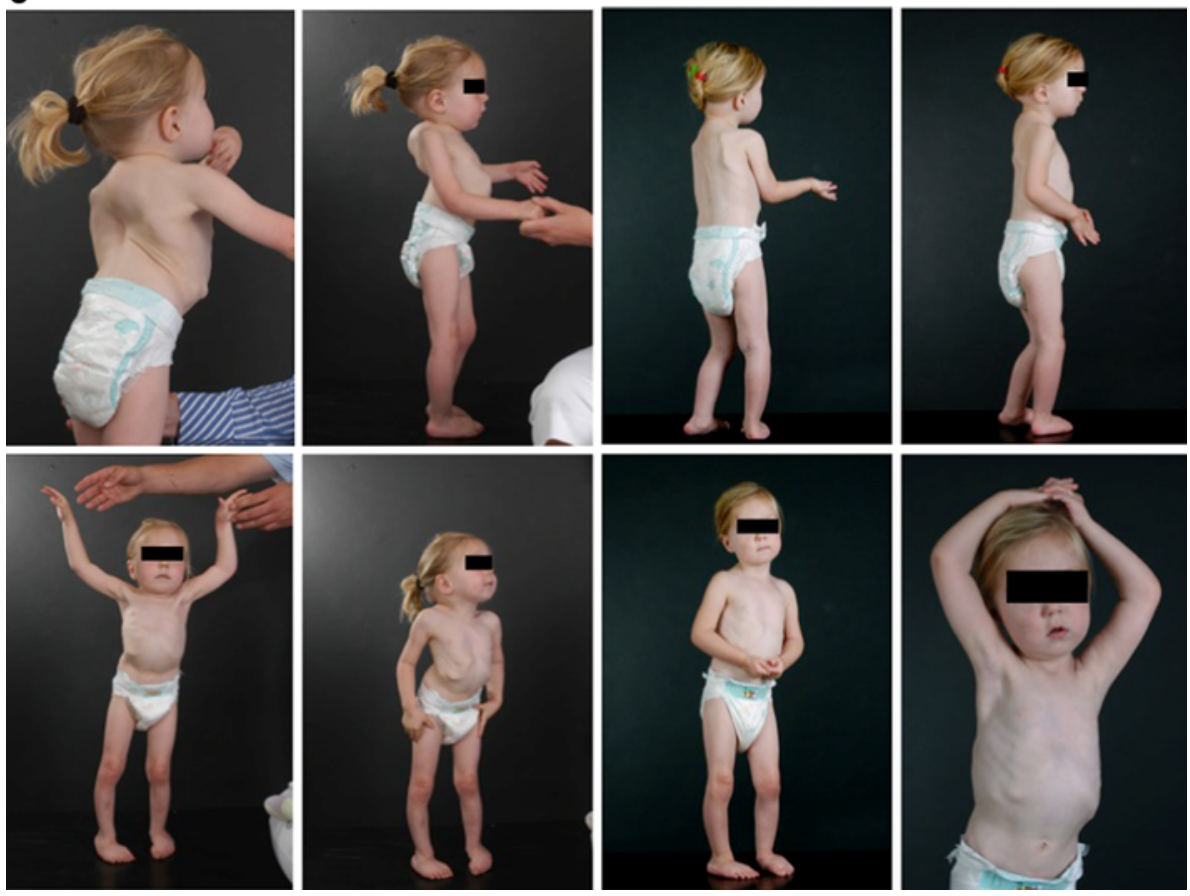
Fig. 2 continued

d
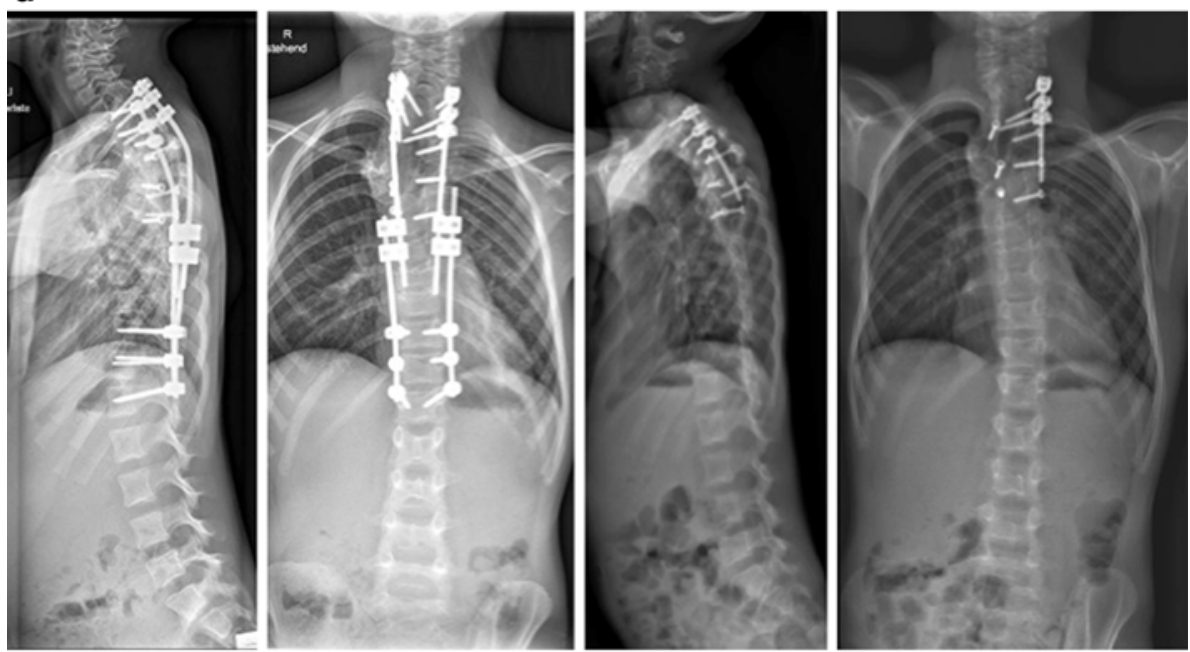

e
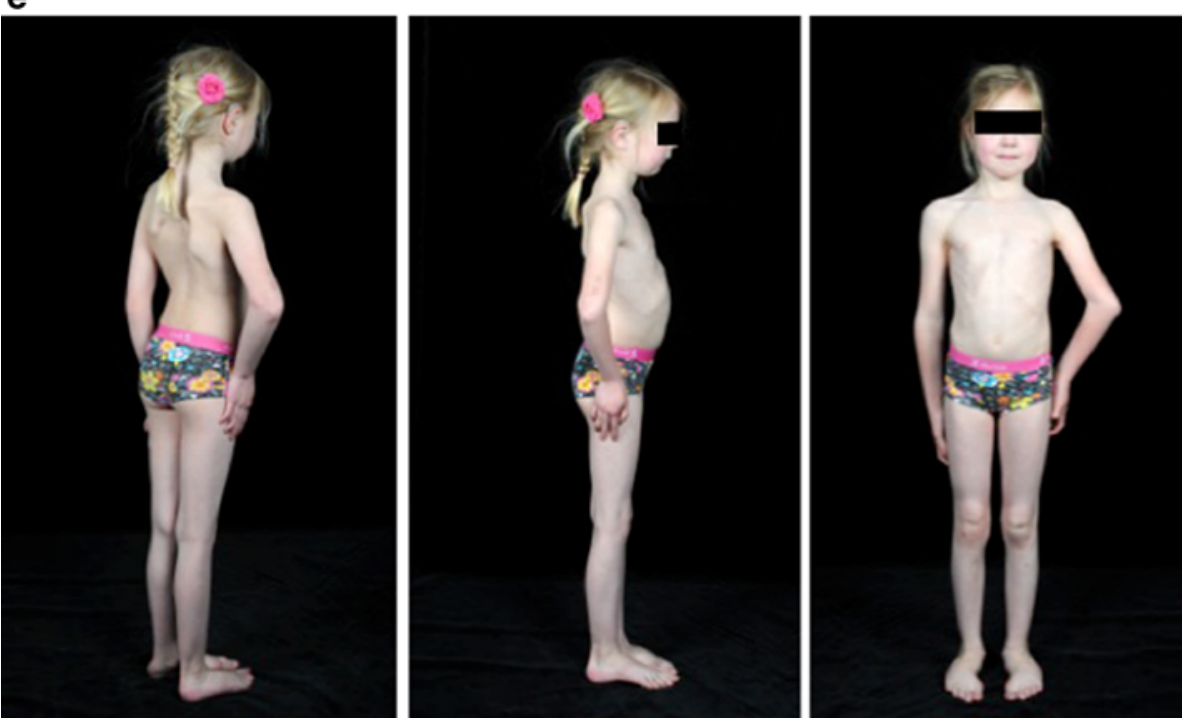

$69^{\circ}$ (range $\left.50-99^{\circ}\right)$ to $29^{\circ}\left(5-44^{\circ}\right)$ with a mean correction of $57 \%(18-92 \%)$. The kyphosis angle was reduced from $126^{\circ}\left(87-151^{\circ}\right)$ to $61^{\circ}\left(54-75^{\circ}\right)$ with a mean correction of $51 \%(44-62 \%)$. At the latest follow-up, the mean scoliosis angle was further reduced to $14^{\circ}\left(5-33^{\circ}\right)$ with a mean correction of $82 \%$ (67-92\%); the kyphosis angle was reduced to $55^{\circ}\left(43-69^{\circ}\right)$ with a mean correction of $56 \%$ (49-65\%).

\section{Secondary curves}

Data for the secondary curves are also shown in Table 1. The mean preoperative compensatory scoliotic curve was $37^{\circ}$ (range $18-54^{\circ}$ ) and was reduced postoperatively to $16^{\circ}$ $\left(9-23^{\circ}\right)$ with a mean correction of $50 \%(17-76 \%)$. At the latest follow-up, the compensatory curve was further reduced to a mean of $7^{\circ}\left(5-11^{\circ}\right)$ with a mean correction of $80 \%(72-87 \%)$.
The preoperative compensatory hyperlordosis was $67^{\circ}$ $\left(44-84^{\circ}\right)$ and was reduced postoperatively to $43^{\circ}\left(37-47^{\circ}\right)$ with a mean correction of $31 \%(-2$ to $53 \%)$. At the latest follow-up, the compensatory hyperlordosis was $54^{\circ}$ $\left(42-79^{\circ}\right)$ with a mean correction of $19 \%(6-42 \%)$.

\section{Complications}

In one patient (patient ML), the preoperatively applied halo crown had to be repositioned due to pin failure. In the same patient, shortly after the primary surgery, proximal junctional kyphosis developed rapidly. Revision surgery for cranial extension of the instrumentation had to be postponed until 40 days after the index surgery due to a cardiac incident during the induction of anesthesia, with asystolia and subsequently successful CPR.

In another patient (patient TJ), PVCR had been performed as a secondary procedure because of insufficient 
stability and loss of correction following his initial surgery 10 days earlier (a $360^{\circ}$ release, Harms cage interposition and pedicle screw instrumentation from T2-T9). This patient presented with a delayed deep wound infection due to a pressure sore over a distal screw, 2 months postoperatively, which was treated by debridement and replacement of the affected screw. Skin closure was carried out over a suction drain with a rotation flap performed by a plastic surgeon. Antibiotic therapy was applied for 6 weeks, and the wound healed without further consequences.

\section{Discussion}

The diagnosis and treatment of EOSD has evolved tremendously in the past decade. While idiopathic early onset scoliosis can be treated both, conservatively and surgically, the treatment of congenital and dysplastic EOSD is mostly surgical, as the problem is an imbalanced growth of the spine and the deformity is often progressive [21]. Mild and moderately severe deformities extending over longer segments can be treated with established methods that do not involve resections or osteotomies (such as isolated posterior growing rod procedures, hemi-epiphysiodesis, and Luque or Shilla techniques [22-24]); however, these procedures are not feasible (technically) or are ineffective in patients with extremely severe and short curves, such as those presented in our series. Some authors would recommend the use of vertical expandable prosthetic titanium rib (VEPTR) [25, 26]; however, if the thoracic deformity is secondary to the spinal deformity, we do not believe that there is any rationale for addressing the secondary chest wall deformity.

The indications for surgical intervention in EOSD are similar to those for adolescent and adult deformities, i.e. curve progression, pain, neurological deficit, deterioration of pulmonary function and malnutrition [27]. In contrast to skeletally mature patients, however, in EOSD, trunk balance plays a lesser role, as most patients are not yet able to walk and they also have the ability to adapt later, as growth and development continue [4]. It is the imbalance of growth of the affected area of the spine, i.e., the deformity per se, that necessitates surgical intervention. In the group of young patients presented here, the indication for surgery was a rapidly progressing, severe angular curvature in a short section of the spine, with or without respiratory failure.

PVCR is an established surgical treatment for severe and rigid spinal deformities over a short region. It is an extensive procedure that is indicated where less-invasive surgical techniques such as SPO and PSO would be insufficient to achieve optimal correction and restore balance. However, it is mostly used in adolescents and adults and its application in young children is not yet well established. For this reason, treatment standards are lacking. Only a few recent studies have included younger patients [12, 14], and in these the main focus was on patients above the age of five. Basically, PVCR in very young children can be compared to the vertebral resection technique described by Bradford and Suk $[8,9]$. However, in our experience there are some small but important differences in the young patient population that should be considered during treatment. These are summarized in Table 3.

In three of the four cases in the present study (patients BA, ML, TJ), halo-gravity traction was applied for several weeks preoperatively for the following reasons: to stretch the abnormally shortened soft-tissues (including vessels, muscles, ligaments, etc.), to decrease the spinal curvature, and to increase the chest volume and thereby improve pulmonary function [28]. In the children with respiratory insufficiency prior to surgery, a marked improvement in the performance of daily activities was observed. Halo-gravity traction also allowed for correction of the compensatory cervical hyperlordosis caused by the hyper-kyphotic thoracic deformity, which facilitated the placement of pedicle screw instrumentation in the upper thoracic spine.

A single posterior approach was used in all cases presented in the study. As highlighted in Table 3, there is

Table 3 Summary of the considerations particular to the use of PVCR in patients with early onset deformities ( $\leq 5$ years of age) as compared with adolescents/adults

- Parameters used in adults for assessment of global spinal balance cannot easily be transferred to pre-ambulatory children

- Surgery as early as possible and with maximal correction at the site of the main deformity prevents the development of non-structural compensatory curves in intact spinal segments

- Preservation of the periosteum away from the VCR site is of utmost importance to prevent unwanted spontaneous fusion

- Long pedicle screw constructs provide sufficient stability but do not influence spinal growth negatively if converted to a growing rod construct later

- The spine and the chest wall are more elastic:

-Allows for sufficient correction of the spinal deformity

-Thoracoplasty is usually unnecessary. Correction of the underlying spinal deformity helps to guide the development of the chest wall

- Conversion to a growing rod construct for the remaining curve should be considered 
rarely any need for an extensive thoracotomy or thoracoplasty, which can otherwise lead to postoperative chest wall scarring, with its lifelong and sometimes irreversible consequences, as seen with VEPTR or the combined approach [9]. This is of considerable importance in these young patients who already have impaired lung function preoperatively.

As opposed to using the common technique of subperiosteal preparation [12], we recommend preservation of the periosteal layer beyond the VCR site, to avoid spontaneous fusion and allow for normal growth. This nonfusion approach also enables the application of a growing rod construct at a later stage, achieving the shortest possible spinal fusion in the end.

In all cases in this series, poly-axial pedicle screws were used because as compared with hooks or wires they offer greater stability and the potential to achieve and maintain correction, with sufficient primary stability [29, 30]. In addition, they allow the control of spinal alignment during the unstable intraoperative phase of the VCR procedure. The concern of some paediatric spinal deformity surgeons, that the use of pedicle screws may lead to spinal canal stenosis, appears to be unsubstantiated [31].

Some surgeons advocate only incomplete correction of the curve, to reduce surgical risks [32]. However, it can be difficult to decide where to stop the correction. Further, it is to be assumed that the best long-term results would be obtained with maximum possible correction of the main deformity, achieving near-physiological spinal curvatures. Our data demonstrate that, with maximal correction, the compensatory scoliotic curves and the hyperlordosis are also corrected substantially, achieving close to physiological values. In children below 5 years of age, with their remaining growth potential, this aspect is of much greater importance than in adolescents.

Some studies have suggested that the shortening of the spine with VCR may be associated with a further decrease in pulmonary function [18]. However, the actual length of the spine is only one factor governing lung function and development: the curvature of the spine, and consequently the volume, configuration and function of the chest wall, should also be taken into account. Early spinal resection (with shortening) and correction of the deformity stops the unbalanced growth of the spine and thorax, and results in an overall lengthening of the anterior trunk during growth. As compared with kyphosis correction using distraction techniques [33, 34], another advantage of shortening the spine is that it is better tolerated by the spinal cord. In this respect, it is similar to resection of a hemivertebra, another standard spine shortening procedure [35].

In contrast to the situation in adolescents and adults, in young children spinal balance is not necessarily the most important factor in spinal deformities. The parameters that describe spinal balance in the adult cannot readily be applied to children under 5 years, because of their different body proportions and because their neurogenic control of posture is still developing [4]. Instead, the severity of the deformity and the rate of progression (imbalance of growth) are of greater importance. In our series, two patients (patients ML, BA) were pre-ambulatory and therefore the notion of spinal balance was not even applicable.

In the series of four patients presented here, there were some early complications associated with the instrumentation or wound healing. However, no spinal cord-related neurological problems occured. Creating an adequate surgical plan is one of the greatest challenges in the treatment of EOSD. The difficulty is that some of these children need preoperative halo traction, and some degree of traction is continued during the surgery too. Due to the altered alignment of the spine, it is difficult to predict what will happen postoperatively when traction is discontinued and the patients are mobilized (upright position). In the case with the early development of proximal junctional kyphosis (patient ML), the correction and alignment achieved during preoperative halo traction and surgery were deemed adequate, as was the length of instrumentation. As discussed earlier, we aim to keep the instrumentation as short as possible, but in this case-in hindsight-it was too short. When developing the surgical plan and determining instrumentation levels, it is important to appreciate the influence of traction on spinal alignment, and especially on the compensatory curves (the main curve being rigid and hence non-responsive to traction).

In the treatment of EOSD, multiple surgeries are common, which differs from the situation in adult or adolescent deformity. In the present series, in all but one patient with longer posterior pedicle screw constructs, subsequent surgeries were necessary after the primary vertebral resection. These involved either conversion to a growing rod construct with multiple lengthenings, or an extension, shortening or modification of the instrumentation, with or without additional osteotomy. This demonstrates that the behaviour of the primary and compensatory curves can be unpredictable in the growing spine, rendering non-fusion instrumentation beneficial, since all options are kept open (Fig. 2).

\section{Conclusion}

The use of PVCR in children with EOSD is limited and conclusions regarding its viability must therefore be drawn with care. It appears to be an effective surgical procedure for the treatment of severe deformities over a short spinal region in very young children, just as it is in adults. 
However, complications may have to be anticipated and there are important differences between its use in skeletally immature children and in older patients. Additional surgical procedures are likely to be necessary in the young, growing spine, and hence non-fusion instrumentation beyond the resection site is advantageous, as it permits spinal growth and the later addition of fusion. The clinical and radiological results of PVCR in the four cases of EOSD presented are encouraging.

Acknowledgment The authors would like to thank Anne F. Mannion for helping to prepare the manuscript.

Conflict of interest None.

\section{References}

1. Dickson RA (1994) Early-onset idiopathic scoliosis. In: Weinstein SL (ed) The pediatric spine: principles and practice. Lippincott Williams and Wilkins, Philadelphia, p 421

2. Campbell RM Jr (2009) Spine deformities in rare congenital syndromes: clinical issues. Spine (Phila Pa 1976) 34:1815-1827. doi:10.1097/BRS.0b013e3181ab64e9

3. Greggi T, Lolli F, Maredi E, Di Silvestre M, Martikos K, Vommaro F, Giacomini S, Baioni A, Cioni A (2012) Surgical treatment for scoliosis associated with rare disease. Stud Health Technol Inform 176:326-329

4. Dimeglio A (2001) Growth in pediatric orthopaedics. J Pediatr Orthop 21:549-555

5. Philips MF, Dormans J, Drummond D, Schut L, Sutton LN (1997) Progressive congenital kyphosis: report of five cases and review of the literature. Pediatr Neurosurg 26:130-143

6. Thurlbeck WM (1982) Postnatal human lung growth. Thorax 37:564-571

7. Emery JL, Mithal A (1960) The number of alveoli in the terminal respiratory unit of man during late intrauterine life and childhood. Arch Dis Child 35:544-547

8. Bradford DS, Tribus CB (1997) Vertebral column resection for the treatment of rigid coronal decompensation. Spine (Phila Pa 1976) 22:1590-1599

9. Suk SI, Chung ER, Kim JH, Kim SS, Lee JS, Choi WK (2005) Posterior vertebral column resection for severe rigid scoliosis. Spine (Phila Pa 1976) 30:1682-1687

10. Boachie-Adjei O, Bradford DS (1991) Vertebral column resection and arthrodesis for complex spinal deformities. J Spin Disord 4:193-202

11. Suk SI, Kim JH, Kim WJ, Lee SM, Chung ER, Nah KH (2002) Posterior vertebral column resection for severe spinal deformities. Spine (Phila Pa 1976) 27:2374-2382. doi:10.1097/01.BRS. 0000032026.72156.1D

12. Lenke LG, O'Leary PT, Bridwell KH, Sides BA, Koester LA, Blanke KM (2009) Posterior vertebral column resection for severe pediatric deformity: minimum 2-year follow-up of thirtyfive consecutive patients. Spine (Phila Pa 1976) 34:2213-2221. doi:10.1097/BRS.0b013e3181b53cba

13. Lenke LG, Sides BA, Koester LA, Hensley M, Blanke KM (2010) Vertebral column resection for the treatment of severe spinal deformity. Clin Orthop Relat Res 468:687-699. doi:10. 1007/s11999-009-1037-x

14. Hamzaoglu A, Alanay A, Ozturk C, Sarier M, Karadereler S, Ganiyusufoglu K (2011) Posterior vertebral column resection in severe spinal deformities: a total of 102 cases. Spine (Phila Pa 1976) 36:E340-E344. doi:10.1097/BRS.0b013e3182015712

15. Leatherman KD (1973) The management of rigid spinal curves. Clin Orthop Relat Res 93:215-224

16. Lenke LG, Newton PO, Sucato DJ, Shufflebarger HL, Emans JB, Sponseller PD, Shah SA, Sides BA, Blanke KM (2012) Complications following 147 consecutive vertebral column resections for severe pediatric spinal deformity: a multicenter analysis. Spine (Phila Pa 1976). doi:10.1097/BRS.0b013e318269fab1

17. Sponseller PD, Jain A, Lenke LG, Shah SA, Sucato DJ, Emans JB, Newton PO (2012) Vertebral column resection in children with neuromuscular spine deformity. Spine (Phila Pa 1976) 37:E655-E661. doi:10.1097/BRS.0b013e318244460d

18. Sponseller PD, Yazici M, Demetracopoulos C, Emans JB (2007) Evidence basis for management of spine and chest wall deformities in children. Spine (Phila Pa 1976) 32:S81-S90

19. Sutter M, Eggspuehler A, Muller A, Dvorak J (2007) Multimodal intraoperative monitoring: an overview and proposal of methodology based on 1,017 cases. Eur Spine J 16(Suppl 2):S153S161. doi:10.1007/s00586-007-0417-8

20. Haschtmann D, Kaiser B, Fekete T, Kleinstück FS, Jeszenszky DJ (2013) Non-fusion approach of growth guidance for the treatment of spinal deformities in young children with a mean follow-up of 4.6 years. In: Global Spine Congress 2013. AO Spine Switzerland, Hong Kong, A195. http://www.global spinecongress.org/GSC\%202013\%20Scientific\%20Program.pdf

21. McMaster M (2011) Congenital Scoliosis. In: Bridwell K, DeWald R (eds) The textbook of spinal surgery. Lippincott Williams and Wilkins, Philadelphia, pp 1118-1138

22. Blount WP, Clarke GR (1949) Control of bone growth by epiphyseal stapling; a preliminary report. J B Jt Surg Am 31A:464-478

23. Marks DS, Sayampanathan SR, Thompson AG, Piggott H (1995) Long-term results of convex epiphysiodesis for congenital scoliosis. Eur Spine J 4:296-301

24. Luque ER (1982) Segmental spinal instrumentation for correction of scoliosis. Clin Orthop Relat Res 163:192-198. http://journals. lww.com/corr/Citation/1982/03000/Segmental_Spinal_ Instrumentation_for_Correction_of.28.aspx

25. Hell AK, Campbell RM, Hefti F (2005) The vertical expandable prosthetic titanium rib implant for the treatment of thoracic insufficiency syndrome associated with congenital and neuromuscular scoliosis in young children. J Pediatr Orthop B 14:287-293

26. Campbell RM Jr, Adcox BM, Smith MD, Simmons JW 3rd, Cofer BR, Inscore SC, Grohman C (2007) The effect of midthoracic VEPTR opening wedge thoracostomy on cervical tilt associated with congenital thoracic scoliosis in patients with thoracic insufficiency syndrome. Spine (Phila Pa 1976) 32:2171-2177. doi:10.1097/BRS.0b013e31814b2d6c

27. Mummaneni PV, Lenke LG, Haid RW (2008) Spinal deformity: a guide to surgical planning and management. Quality Medical Publishing Inc., St. Louis

28. Sink EL, Karol LA, Sanders J, Birch JG, Johnston CE, Herring JA (2001) Efficacy of perioperative halo-gravity traction in the treatment of severe scoliosis in children. J Pediatr Orthop 21:519-524

29. Liljenqvist U, Hackenberg L, Link T, Halm H (2001) Pullout strength of pedicle screws versus pedicle and laminar hooks in the thoracic spine. Acta Orthop Belg 67:157-163

30. Suk SI, Lee CK, Kim WJ, Chung YJ, Park YB (1995) Segmental pedicle screw fixation in the treatment of thoracic idiopathic scoliosis. Spine (Phila Pa 1976) 20:1399-1405

31. Fekete TF, Kleinstuck FS, Mannion AF, Kendik ZS, Jeszenszky DJ (2011) Prospective study of the effect of pedicle screw placement on development of the immature vertebra in an in vivo 
porcine model. Eur Spine J 20:1892-1898. doi:10.1007/s00586011-1889-0

32. Dickson RA (2001) Early-onset idiopathic scoliosis. In: Weinstein SL (ed) The pediatric spine: principles and practice. Lippincott Williams and Wilkins, Philadelphia, pp 321-328

33. Dabney KW, Ehrenshteyn M, Agresta CA, Twiss JL, Stern G, Tice L, Salzman SK (2004) A model of experimental spinal cord trauma based on computer-controlled intervertebral distraction: characterization of graded injury. Spine (Phila Pa 1976) 29:2357-2364
34. Jarvis J, Strantzas S, Lipkus M, Holmes L, Dear T, Magana S, Lebel D, Lewis SJ (2013) Responding to neuromonitoring changes in three column posterior spinal osteotomies for rigid pediatric spinal deformities. Spine (Phila Pa 1976). doi:10.1097/ BRS.0b013e3182880378

35. Ruf M, Jensen R, Jeszenszky D, Merk H, Harms J (2006) Hemivertebra resection in congenital scoliosis-early correction in young children. Z Orthop Ihre Grenzgeb 144:74-79. doi:10. 1055/s-2006-921417 